\title{
Hypoplastic left heart syndrome surgical treatment augmented with phenoxybenzamine
}

\begin{abstract}
Hypoplastic left heart syndrome (HLHS) is a rare congenital heart defect in which the left heart is severely underdeveloped. In recent years, HLHS has undergone major changes in the way it is diagnosed and treated; and its prognosis. Prior to recent treatments and surgical procedures, HLHS was fatal. Today, it is expected that neonates affected by this syndrome, who undergo modern surgical procedures, have a $70 \%$ chance of reaching adulthood. ${ }^{3}$ HLHS is usually treated through surgeries which are performed in three different stages: the Norwood procedure, the Glenn procedure and the Fontan procedure. The purpose of these surgeries is to create a way for the blood to flow in and out of the heart more normally so that the neonate body can receive adequate amounts of oxygenated blood. This paper will focus mainly on the Norwood procedure and treatment with phenoxybenzamine shortly after the surgery. Perioperative management is recommended before surgery. It is reported that administering phenoxybenzamine-which is a vasodilator-can reduce early mortality. It is further reported that phenoxybenzamine reduces afterload of the single ventricle and produces more stable parallel circulation by ameliorating the postoperative fluctuations in systemic vascular resistance. ${ }^{1}$ This article will discuss the pharmacology of phenoxybenzamine, surgical and physiological implications of the Norwood procedure, and the pros and cons of administering phenoxybenzamine during such procedure.
\end{abstract}

Volume 9 Issue I - 2017

\author{
Awok M Majok,' Nicholas A Kerna, ${ }^{2}$ Tony \\ Brown ${ }^{3}$ \\ 'College of Medicine, University of Science, Art and Technology, \\ USA \\ ${ }^{2}$ Major General Hugh G. Robinson Center for Medical Studies, \\ USA \\ ${ }^{3}$ Harvard University, USA
}

Correspondence: Nicholas A Kerna, College of Medicine, University of Science, Art and Technology, 7583 Sourdough Dr, Morrison, CO 80465, USA, Email Nicholas.kerna@usat.edu

Received: May 26, 2017 | Published: May 29, 2017

Keywords: hypoplastic, neonate heart, norwood procedure, phenoxybenzamine

Abbreviations: CPB, cardiopulmonary bypass; HLHS, hypoplastic left heart syndrome; IV, intravenous; PVR, pulmonary vascular resistance; SVR, systemic vascular resistance

\section{Introduction}

Before these new procedure became available, comfort care was the only option for these neonates. When neonates with HLHS undergo the three-step Norwood procedure, they are expected to live to adulthood with a single ventricle supplying all the blood needed for the pulmonary and systemic circulations. They also end up depending on a shunt to supply blood between the pulmonary arteries and the aorta. Though these procedures are remarkable, they still have a high mortality rate because the cardiac output is decreased. There is also an imbalance between the pulmonary and systemic circulation which can result in a sudden collapse of the heart. ${ }^{1}$ Because of the high mortality rate, many surgeons are opting for treatment with vasodilators, like phenoxybenzamine, postoperatively. The goal of treatment with phenoxybenzamine is to balance the circulation by reducing systemic vascular resistances (SVR) so that the neonates can have higher systemic blood flow as opposed to pulmonary blood flow. Ever since phenoxybenzamine has been tried postoperatively, however, there has been no significant research reported on its effectiveness. This article discusses the basic pharmacology of phenoxybenzamine, and physiological and surgical results of its use with the Norwood procedure. ${ }^{1}$

\section{Discussion}

Phenoxybenzamine is a vasodilator that works on smooth muscle. It blocks both alpha-1 and alpha-2 adrenergic receptors, but works better on alpha-1. It is lipid soluble so there is a high chance that it will be absorbed in fatty tissues if given in large dosages. Also, fat soluble medications tend to have a long half-life; hence, one of the reasons they accumulate in fatty tissues. Caution should be taken with pregnant women, obese patients and babies since they each tend to have high amounts adipose tissue. If taken in large doses, phenoxybenzamine can accumulate in brain and liver tissues causing neurological issues, like seizures; and liver complications.

Phenoxybenzamine is considered a slow-acting medication with a half-life of six hours or less. It reaches its peak an hour or more after its intravenous (IV) administration. The dosage and route of administration of this medication determines its action as a long-acting or short-acting medication. The main action of phenoxybenzamine is systemic vasodilation due to its direct blockade of alpha-adrenergic receptors in the vascular smooth muscle. The major hemodynamic effect is a noticeable decrease in SVR and central venous pressure along with a compensatory increase in cardiac output. ${ }^{1}$ When the blood flow in the coronary artery, the resulting effect is an increase in cardiac performance.

Phenoxybenzamine increases pump flow rates during cardiopulmonary bypass (CPB) thereby decreasing metabolic acidosis after surgery. Phenoxybenzamine is also more effective than sodium nitroprusside in improving perfusion in the tissue after the bypass. ${ }^{1,2}$ Though physicians and researchers have not reached a consensus, still many have opted to administer phenoxybenzamine with the Norwood procedure because of its vasodialating effects.

Historically, several surgical strategies were attempted in treating HLHS with no great success. Norwood and colleagues were the first to achieve a successful surgical procedure in the 1980s. ${ }^{3}$ The Norwood procedure is a three-stage surgical procedure that is used to create an atrium that will deliver pulmonary venous blood from the right ventricle to the aorta and coronary arteries for a circuit for systemic flow in HLHS. ${ }^{1}$ The purpose of the procedure is to allow the right ventricle to be the only pump. During surgery the main pulmonary artery and the aorta are connected. Once they are connected, the pulmonary artery is then shut off from the two branching pulmonary 
arteries that direct blood to the lungs. Once the pulmonary artery is shut off, a shunt is created between the aorta and the pulmonary arteries in order to supply blood to the lungs. ${ }^{4}$ It is ideal to perform the first stage of the Norwood procedure immediately after birth. There does remain variability in practice among providers regarding the Norwood procedure. 5

Research indicates that early death in neonates after the Norwood procedure is connected to over circulation of the neonate's system. Therefore, the goal is to try and balance the circulation by increasing pulmonary vascular resistance (PVR). This is accomplished by inducing hypercapnia or limiting inspired oxygen before and after the surgery. Even though the Norwood procedure seems to be a good treatment option for HLHS, the neonates have higher risk of pleural effusions. This effusion may require these newborns to be put on long-term chest tube; modified diet; replenishing their serum proteins; medications; and more procedures, resulting in many other complications. The newborns that develop pleural effusions following the procedure are expected to have long hospital stays which puts them at a greater risk of acquiring nosocomial infections, such as MSRA and pneumonia. Therefore, it is imperative that surgeons and staff caring for these patients pay close attention to any contributing factors that might cause pleural effusion.

\section{Conclusion}

There was once a time when the diagnosis of HLHS was a death sentence to infants. ${ }^{6}$ Newer surgical procedures for HLHS have resulted in a better prognosis for patients, many living until and through adulthood. ${ }^{3}$ Treatment with the Norwood procedure allows patients to get oxygenated blood into their systems. With the vasodilator, phenoxybenzamine, the systemic and pulmonary circulation can be balanced ensuring enough blood and oxygen supply. Since phenoxybenzamine is fat soluble and can accumulate and stay in the body longer, it can cause dangerous side effects, such as seizures. Therefore, caution should be observed. The Norwood procedure is effective at prolonging the lives of neonates affected by HLHS. By creating a shunt that allows the delivery of oxygenated blood into the system, neonates can be expected to live longer. Since the right ventricle is the only working part or pump for these patients, caution is advised as it can become overworked. If the right ventricle gets overworked, then it will not be able to perform all the functions necessary to supply blood and oxygen. It is in the best interest of these patients not to participate in extraneous exercises when they reach adulthood. Treatment of HLHS via the Norwood procedure with the addition of phenoxybenzamine appears efficacious in balancing the affected infant's circulation, thus contributing to improved prognosis and prolonging life.

\section{Acknowledgments}

None.

\section{Conflicts of interest}

Author declares there are no conflicts of interest.

\section{Funding}

None.

\section{References}

1. Guzzetta NA. Phenoxybenzamine in the treatment of hypoplastic left heart syndrome: a core review. Anesth Analg. 2007;10 (2):312-315.

2. Motta P, Mossad E, Toscana D, et al. Comparison of Phenoxybenzamine to Sodium Nitroprusside in infants undergoing surgery. J Cardiothorac Vasc Anesth. 2005;19(1):54-59.

3. Feinstein JA, Benson DW, Dubin AM, et al. Hypoplastic left heart syndrome: current considerations and expectations. J Am Coll Cardiol . 2012;59(1 Suppl):S1-42.

4. Kitahori K, Murakami A, Takaoka T, et al. Precise evaluation of bilateral pulmonary artery banding for initial palliation in high-risk hypoplastic left heart syndrome. J Thorac Cardiovasc Surg. 2010;140(5):1084-1091.

5. Wernovsky G, Ghanayem N, Ohye RG, et al. Hypoplastic left heart syndrome: consensus and controversies in 2007. Cardiol Young. 2007;17(2 Suppl):75-86.

6. Maher KO, Gidding SS, Baffa JM, et al. New developments in the treatment of hypoplastic left heart syndrome. Minerva Pediatr. 2004;56(1):41-49. 